Withering Waits: The Development of a Referrals Management System within a Clinical Psychology and Counselling Service

Jon Willows ${ }^{1}$, Robert Marsh ${ }^{1}$, Sarah Gasson ${ }^{2}$,

${ }^{1}$ Sussex Partnership NHS Trust, Brighton

${ }^{2}$ Canterbury Christchurch University 


\title{
Withering Waits: the Development of a Referrals Management System within a Clinical Psychology and Counselling Service
}

\author{
Jon Willows, Robert Marsh, Sarah Gasson
}

\begin{abstract}
This paper charts the implementation and development of a referrals management system within a secondary care National Health Service (NHS) clinical psychology and counselling service over a period of approximately three years. The new system that is described has brought about dramatic reductions in numbers waiting for both assessment and therapy, as well as modest improvements in attendance rates at first assessment. Other changes have resulted from the new system, such as a more unified, transparent and collaborative approach, a move to more of a consultation model of assessment and an expansion in the breadth of clinicians' roles. The paper explores the challenge of having to continue to meet a demand that far outstrips the available resources. This raises dilemmas in clinical decision-making, in the positioning of the department in relation to referrers, and in maintaining an efficient service with minimal waiting lists without losing the depth and variety of work that are strengths of the department.
\end{abstract}

Keywords: Referrals, Waiting lists, Psychological therapies, National Health Service.

\section{National picture}

The reduction of waiting times has been a continuous Government priority. The NHS Plan (Department of Health 2000) includes a commitment to reduce waiting times - the target for a first outpatient appointment being a maximum of 12 weeks (Department of Health 2004). Whilst there has been general decrease in overall waiting times, waiting lists for psychotherapy services remain extensive (Department of Health 2004). At least half of psychological therapy services surveyed in working age adult mental health services reported a wait of between 10 and 23 months between referral and the start of therapy (Rezin and Gardner 2006). There are multiple causes for long waiting lists, including demand exceeding supply, poor management of waiting lists, inappropriate referrals and higher nonattendance rates in mental health and psychotherapy services than the average across all specialties (Hughes 1995; O'Loughlin 1990).

\section{History of the local service}

The provision of secondary and tertiary psychological therapies in Brighton and Hove is perhaps unusual in continuing to be provided for the time being largely through out-patient, psychological therapy services rather than via multi-disciplinary teams. Until January 2004, the Brighton and Hove Department of Psychology and Counselling (where the authors are based) operated a sectorised system. Each 'sector' psychologist was responsible for a geographical area of the city and each had a separate waiting list for assessment and therapy. Whilst this approach had some strengths - for 
instance clients could generally see the same psychologist for therapy whom they had seen for assessment - it also had many drawbacks. Sector psychologists also often felt personally burdened and responsible for the waiting times, whilst being unable to reduce them. The range of therapeutic approaches available to clients was also restricted by the particular approach of each psychologist. A 'postcode lottery' also meant that clients could wait substantially longer in some sectors than in others.

\section{Introduction of the referrals management system}

\section{Development of single point of referral}

A new system was introduced in 2003 which sought to address these problems and to reduce waiting times. This replaced geographical sectors with a single point of referral through which all secondary and tertiary referrals into the department were processed. A new post of referrals manager was established and held by a clinical psychologist within the team. The referrals manager read all new referrals, gathered additional referral information where necessary, and made initial decisions about suitability. Consequently, a significant number of referrals were re-directed to other local services where clients could often be seen more quickly whilst also accessing an appropriate service. Several new initiatives were developed to support this process, as described below.

\section{Referral criteria}

Explicit referral criteria were developed giving the referrals manager a clear and transparent basis upon which to make decisions about client suitability. Copies were sent to all referrers indicating those people most likely to benefit from the service. These guidelines were linked to the Global Assessment of Functioning Scale (GAF) (APA 1994: 34). We suggested that GAF scores of between 21 and 50 were likely to be suitable for secondary and tertiary psychological services. By contrast, scores of between 51 and 70 were considered likely to be suitable for the primary care counselling service (PCC). For all referrals not meeting the criteria, a letter was sent to the referrer containing our referral criteria and a list of key alternative local services.

\section{Pre-assessment questionnaire}

A pre-assessment questionnaire (PAQ) was sent out to clients who, from the referral information, appeared to meet our criteria. This was in two parts. Part A asked about demographics, preferred contact details, the involvement of other professionals, risk issues and about any children living with the client. It also provided space for the client to highlight any concerns or particular issues that we might need to consider (such as access requirements or specific anxieties about attending). Part B asked about presenting problems, what had helped in coping with them, current relationships, occupational issues, drug and alcohol use, medication, previous therapy and what the client hoped to gain from the service. Whilst this part of the questionnaire was optional, most chose to complete it.

In considering which questions to include, we tried to gather information that would be informative without being overly intrusive since, at that early 
stage of assessment for suitability, there was no guarantee that the process would go further. With this in mind, there were no specific questions about family history or traumatic events in childhood.

One of the functions of the questionnaire was to assess motivation. Consequently, it was necessary to return Part A by a specified date in order to continue with the assessment process. If we received any information in the referral letter suggesting that the prospective client may not be able to read the questionnaire, for whatever reason, then the questionnaire was not sent. One possible criticism of this procedure is that it risks excluding some of the less literate or more chaotic clients.

The questionnaire allowed us to re-direct clients who could be helped by another service without going through the process of further assessment. For the majority who went on to attend assessment sessions, the questionnaire also provided helpful background information for the assessing clinician. Finally, we sent a Clinical Outcomes in Routine Evaluation - CORE (Core System Group 1998) with the questionnaire, which was helpful in identifying risk issues that might need prompt attention.

\section{Allocating assessments}

Upon receiving returned questionnaires, the referrals manager allocated particular assessments to particular clinicians. A team consisting of clinical psychologists, counselling psychologists and counsellors possesses a broad range of skills and experience. A major advantage of having one team member allocating assessments is that consideration can go into establishing the likely best 'fit' between client and assessor so that the service can remain responsive both to clients' needs for clinicians with particular expertise as well as to clinicians' needs for certain cases for their own development and also that of any trainee clinical psychologists alongside them.

\section{Client choice}

If, based upon responses to the questionnaire, the service seemed suitable for the client, a letter was sent inviting them to choose an appointment time and date. Previously there was often no choice but the new system usually allowed for some choice of time, date or location in line with government recommendations at the time.

\section{Audit}

Diagnostic audit codes were developed allowing assessors to indicate the client's main issues and to indicate severity using the GAF. Likewise, CORE forms given with the pre-assessment questionnaire, complemented outcome measurement. An audit of the pre-assessment questionnaire suggested staff generally saw it as a helpful assessment tool. Interestingly, it seemed to be used in different ways according to the therapeutic approach of the clinician. For instance, more psycho dynamically oriented therapists tended to highlight the process of how the form appeared to have been completed and those with a more solution-focused orientation made more use of highlighting the resources people had identified as helping 
them to cope. The feedback provided by clients enabled us to clarify and amend some of the questions in the questionnaire. It also made us more aware of the way in which some had been completing the CORE strategically in order to try and elicit a more active response from us.

\section{Results - impact of new system}

\section{Numbers waiting}

Figure 1 below, shows that the number of people waiting for assessment has dropped from 510 to around 20. Since the new system has been operating there has been a reduction in those waiting for assessment of some $95 \%$. This has continued to remain stable over time because the new system allows for the flexible allocation of clinical time to either assessment or therapy; depending on where need is greatest. The wait for a first assessment has dropped from 18 months, in one of the old sectors, to approximately 16 weeks, and sometimes considerably less than that.

\section{Figure 1. Assessment waiting list}

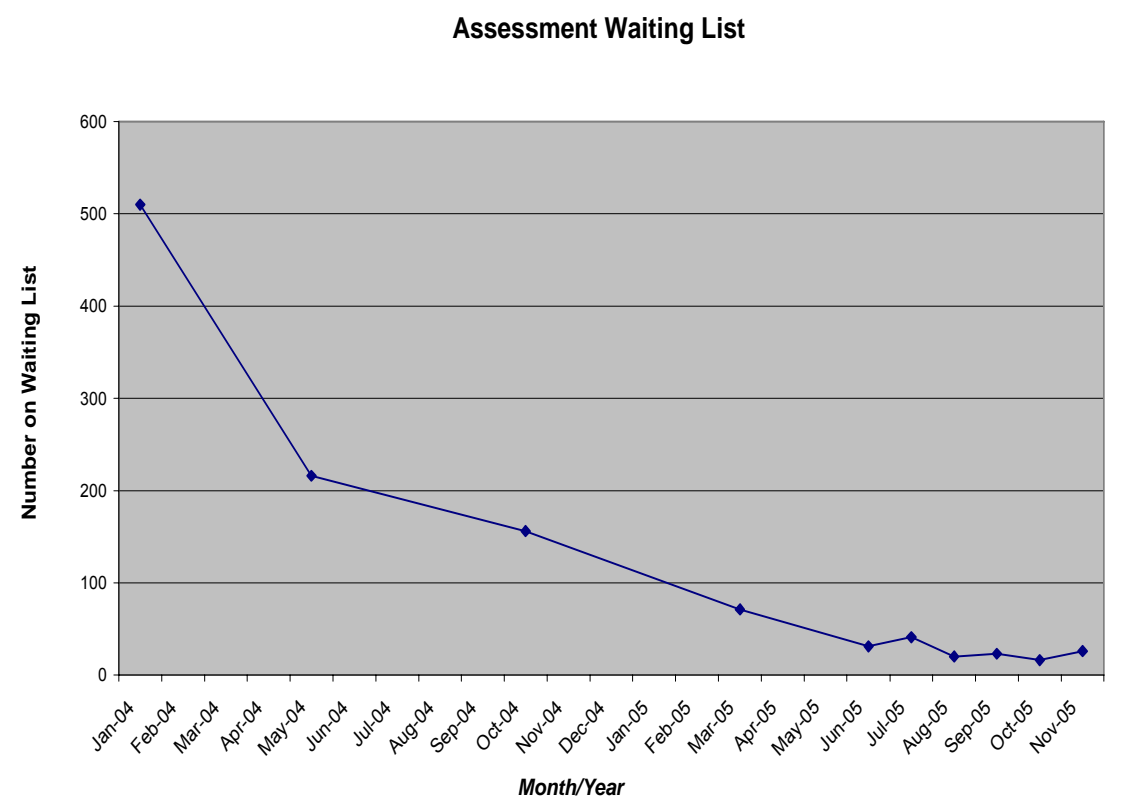

As indicated in Figure 2 below, there have been similar decreases in the number of people waiting for therapy - from 280 to 86 . This number has continued to fall and at the time of writing was less than 50. Encouragingly, although the waiting time for therapy remains long, this is gradually reducing. If the therapy list continues to decline at the same rate as it has done over the past year, there should be no waiting list within a few months. 
Figure 2. Therapy waiting list

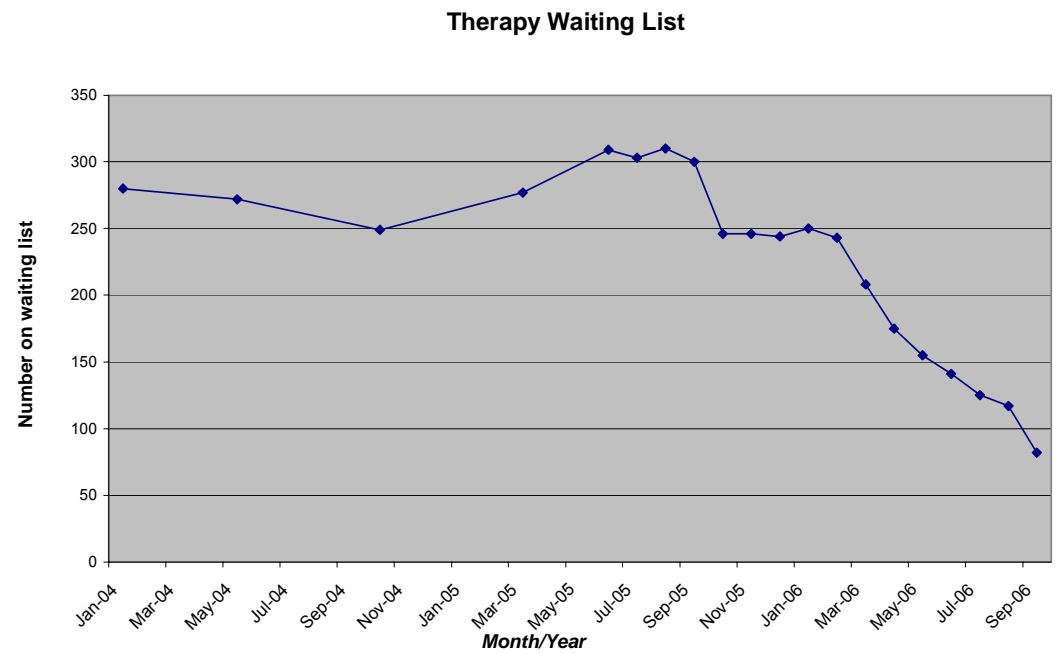

\section{Referral rate}

The number of new referrals received in the department has also decreased under the new system (figure 3). This number continues to fall. Based on our transparency with regard to waiting times, referrers now have a very clear idea of how long clients may have to wait for psychological therapy. Similarly, with regular liaison and by repeatedly sending out referral criteria alongside information about other services, some referrers appear to begun to develop a much more informed view about suitability for this service.

Figure 3. Referrals received per month from January 2004 to November 2005

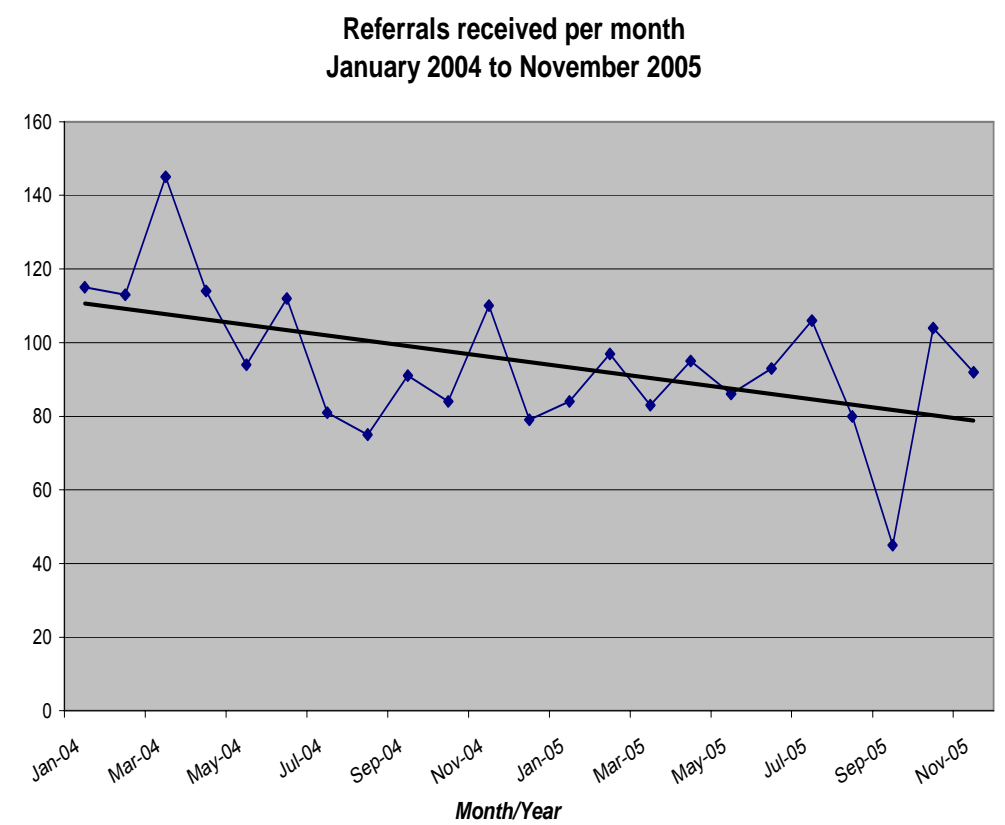




\section{DNA rates}

The number of assessment appointments missed with out notice (DNA) has seen a small but important reduction (Figure 4). This may have been due to the greater choice now offered to clients for their assessment appointment. It is also likely to have been due to the introduction of several new gateways in the process from referral to first assessment at which the suitability of the service for the person is assessed and motivation explored.

In previous studies, the effect on attendance rates from sending opt-in forms and questionnaires has been mixed. For example, Markman \& Beeney (1990) and Ambrose \& Ormond (1996) found no significant effect on DNA rates for initial appointments from sending an opt-in form with an information leaflet, or a ten page questionnaire, respectively. However, Eynon (1993) reported that the use of a psycho dynamically oriented preassessment questionnaire helped reduce DNAs. Various forms of client opt-in, whether by asking client to send in a return slip indicating intention to attend (Anderson \& White 1994; Green \& Giblin 1988), asking them to telephone (Chiesa, 1992) or offering a choice of appointment together with asking for confirmation of attendance (Reid \& Mclvor 2005) all reduced non-attendance rates. Amongst the waiting list strategies listed in the paper 'Organising and delivering psychological therapies' (Department of Health 2004) are opt-in procedures via contacting the service for an appointment and opt-in procedures via questionnaire.

Figure 4. Assessment DNA (\%) per month from January 2002 to November 2005

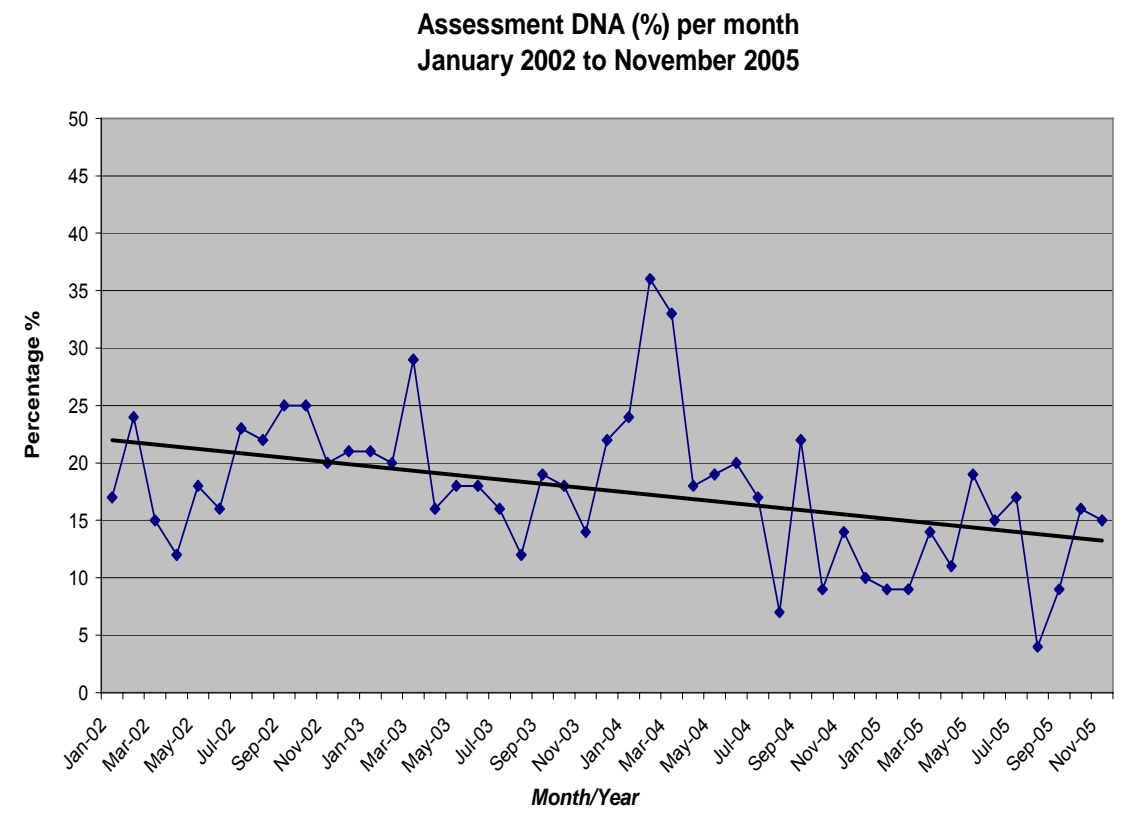




\section{Transparency}

When operating a number of semi-autonomous sectorised systems, clinicians, for many reasons, could be somewhat reserved about their waiting lists. Under the new system, the team shared the same waiting list. This appeared to prompt more openness and a shared sense of ownership amongst the team. It was then possible to extend transparency about the waiting time incurred on this central list to clients and referrers.

Accepting the reality of long waits has clearly been a source of distress for referrer, client and assessor alike. However, as a result we are much clearer about the actual capacity of the service to see clients and have been able to convey that clearly and confidently to referrers. Subjectively, this seems like a much healthier process in which clinicians do not need to feel driven by the illusion that working 'ever harder' will somehow make the waiting list disappear. Nor is the reward of the work undermined by the guilt behind erroneously promising a service that could not realistically be offered in the foreseeable future.

\section{Working with referrers and managing change}

This process has positioned the referrals manager in much closer contact with referrers. This has been an illuminating process in itself. It has brought old tensions to light and provided the opportunity for working more actively with inter-service conflict in a system where open communication, for many reasons, can be less than ideal.

On occasion, our greater transparency about waiting times met with anger and complaint. Likewise meeting with Community Mental Health Teams has, on occasion, tended to highlight old struggles and the enduring status of stereotyped notions of clinical psychology. It also became clear that many referrers had not developed an in-depth knowledge of where best to refer. The tried and tested referral pathways have been difficult to change and, rather than seek guidance, some might continue to follow these wellworn routes using psychology as a triage service. In some quarters, use of our criteria has also had limited uptake. However, we accept that the prospect of familiarisation with yet another complex set of criteria is unlikely to appeal to a busy GP.

Alongside these tensions, the increased contact with other services has led to a degree of rapprochement. For example, some GP practices have responded very well to letters informing them that the service is struggling with a significant backlog of cases. When referrers know just how much demand exceeds capacity, it invites some understanding and promotes the case for change. GPs have also welcomed the opportunity to meet to discuss referral criteria and to contribute to conversations in which a local 'map' of psychological services is being established.

\section{Working with clients' reactions}

Although the wait for a first assessment appointment is now within more acceptable limits, the waiting time for therapy has not yet reflected the major reductions in numbers of people waiting for therapy. When the likely 
waiting time is years rather than months, the process of assessment becomes the focus for some particularly difficult feelings.

For clients, understandably, this can involve disappointment, shock and anger. Being made aware of a wait of several years for therapy, when one's hopes are high, can be a powerful trigger to the resurgence of underlying feelings of worthlessness or abandonment at the point of need. Indeed, the experience of assessors suggests that much of the work in recent assessment sessions has been around exactly these issues. The effect upon staff has also been considerable. The rigours of working through these emotionally charged issues have been a central feature of much discussion and peer support.

\section{Moving towards a 'consultation' model}

There has been a shift in thinking away from treating 'assessment' as an automatic conduit to a place on the therapy waiting-list. Instead, the sessions have increasingly been used as discrete consultations in their own right. The experience of the team has been that that this process could be a powerful intervention in itself, which has mostly been accepted and welcomed by both staff and clients. As always, our aim was that the client should feel heard and understood. In providing a consultation, our goal was that they should also receive a useful formulation and ideas about services and resources that could be mobilised relatively quickly.

Typically, the options ranged from recommending more of what had worked already (e.g. making use of trusted sources of support, exercise, relaxation) to signposting reading material, recommending psycho-educational or selfhelp groups, advice services (e.g. supporting a return to voluntary or paid work, or housing advice), primary care counselling, or approaching other providers of psychological therapy. This change to a more consultative and indirect role was difficult to establish since it required a different kind of thinking and an adaptation to a different kind of service. Recognizing that many clients could benefit from some form of very brief intervention elsewhere could be a difficult shift, especially for those of us accustomed to accepting people onto our own therapy waiting-list. A more consultative practice could also, perhaps, challenge hidden fantasies of omnipotence on the one hand and arouse strong guilt feelings on the other. More generally, it represented a marked shift away from the more traditional emphasis on direct clinical work.

\section{Changing traditional ideas of what the service offers}

Introducing the new system saw changes in the balance of workloads of many psychologists in the department away from largely direct clinical work and towards more indirect clinical activity. This involved reconfiguring resources and investing significant time in the process. For instance, establishing the referrals manager post meant relinquishing the equivalent of at least one half-time clinical post. Referrals management meetings also require staff to redirect some clinical time to the referrals process. Nevertheless, Figure 5 (below) counters the hypothesis that if we were simply to do more direct clinical work, rather than actively manage the referrals process, we would reduce the waiting list. In fact, the new system has been able to almost completely eliminate the assessment waiting list 
(as well as dramatically reducing the therapy waiting list), despite clinicians now offering far less assessment appointments.

\section{Figure 5. Assessment appointments offered per month}

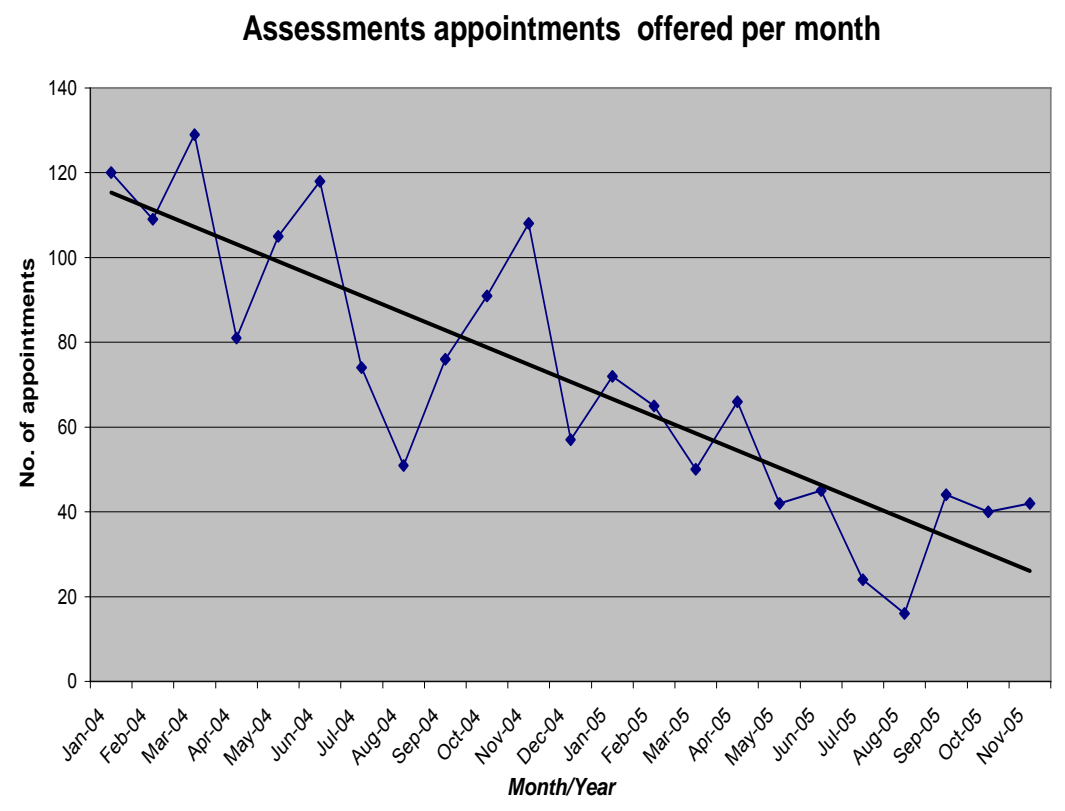

Many clinicians now perform a greater breadth of work, such as developing specialist services, systems management, clinical supervision, consultation, and inter-service liaison. There has also been more engagement with GPs, $\mathrm{CMHTs}$, and other mental health services. This makes the work more varied and interesting, reduces the likelihood of burnout, and makes use of the breadth of clinical psychology training. The team has spent more time together and has been able to appreciate one another's ways of working. This has been reflected in more joint sessions, referrals management group discussions, team-building days, reflective groups and presentations on areas of specialist interest. This new feel to the department, further diversified and enriched by new staff with different skills, has been liberating even though previous role identifications have had to be modified.

Whilst these changes have been positive, it is has also been important to ensure that many of the valuable aspects of the previous identity of the department were not been lost. Historically, the department has created a solid psychotherapeutic base through creating several specialist therapy services and investing in extensive continued professional development for staff (CPD). It contains clinicians who are experienced in delivering both a breadth and depth of therapeutic approach which has enabled it to offer effective medium to long-term interventions to people with significant difficulties. To have thrown this rich 'bath water' out in the interests of producing efficient 'short-term only' babies would have been a sad loss for clients and clinicians alike. 


\section{Towards the future}

\section{An overview of maintenance and development}

At the time of writing, the referral rate and the number of people waiting for a service have both declined and continue to fall. Consequently, we have begun to implement maintenance strategies to make best use of the skills in the department.

Our experience suggests that the following factors are likely to be important in the long term:

- Accurate assessment of the client's needs and suitability for the service

- Accurate identification of the place of the service on the 'map' of local psychological therapies

- Promotion of co-working with other agencies

- Staying up-to-date on local service context and alternative referral pathways

- Staying up-to-date on government recommendations and current clinical guidelines

- Making best use of the shifting skills base within the team

- Balancing the above with CPD needs and individual preferences

In order to maintain and develop this situation, we also recognise that the following issues will need to be addressed.

\section{Service capacity}

It is clear that an accessible service has to work within its resources. Inevitably this involves setting quotas so that demand does not outstrip resource in a way that creates an unacceptably long waiting-list. Realistic quotas must be based upon an accurate appreciation of service capacity. We established this by asking clinicians to estimate the amount of time they dedicated to direct clinical work given the broad range of activities they might undertake in an average week. We found that the actual direct clinical time available was about half of the total work hours. Having established an average treatment length of, in this case, 30 sessions, it was possible to estimate how many clients could be treated per year. In our case, demand (in terms of referrals per month) outstripped actual capacity by a ratio of $16: 1$. This baseline figure might then be used to regulate the number of referrals accepted and to support a case for acquiring more resources.

\section{Dilemmas of inclusion and length of therapy contract}

In a situation in which demand repeatedly outstrips resource, the risk is that certain client groups, who are perhaps either too complex or else too 'straightforward' for time-limited secondary care psychological therapy, may be unable to access the service. It may be that the most depressed, those least familiar with local systems (such as refugees and asylum seekers) and the least literate, find it most difficult to access a system that requires one, if not two, layers of opting-in. 
Another potential casualty may be the depth of work we undertake. For instance, one suggested solution has been to adopt a primary care model to secondary care work and to offer a fixed and small number of sessions as a maximum, irrespective of presentation. Not only might this serve to propel the 'revolving-door' of repeated referrals, it may not be favoured by many clinicians who, for instance, have acquired extensive experience and expertise in longer-term work. In our experience, as the severity threshold for the psychological services has risen, the complexity of the work has increased; consequently the length of time necessary to work with clients to achieve meaningful results has grown. Inevitably, this leads to a reduction in the actual number of clients the service can see each year whilst avoiding a waiting list.

\section{Communication}

It is essential that communication with referrers continues to be clear and helpful. Regular liaison with CMHTs has been shown to lead to better referrals (Rezin and Gardner, 2006). Referrers need to have the relevant information to enable them to make good referral choices earlier on, and to have a better understanding of the way that psychological therapy services work. Perhaps even more important is the development of sound relationships with referrers so that we can take a collaborative position given our shared perspective on the imbalance between resources and demand.

One idea is to provide referrers with a newsletter containing up-to-date waiting-time information and descriptions of what the service provides. Another idea (provided by a referrer) is to produce a 'map' on a single piece of A4 (to promote uptake) to distinguish different services, their suitability criteria, referral pathways along with signposts to more detailed information about a particular service including a contact telephone number. Simply discussing the idea has meant that important inter-service conversations have taken place providing the opportunity to sort out long-standing assumptions and misperceptions of 'who does what with whom'.

\section{Pragmatic or clinical decision-making?}

We are aware that, in avoiding developing another long waiting list, our solution involves imposing manageable limits on access to longer-term individual therapy. Within these limits it is clearly contingent upon us to be appropriately selective and to make responsible use of very scarce state resources. However, the accounts we share amongst ourselves about our decision-making often reveal a difficult tension between clinical need and financial management. On the one hand, our decisions not to take some people on for therapy are clearly justifiable because a traditional psychological therapy approach is unlikely to help everyone. Many clients could also benefit from consultations, brief therapy or other communitybased services. We also recognise that therapy is not a modern panacea and that there is evidence to support our view that brief interventions can also be effective. More philosophically, we can also comfort ourselves with community psychology and social constructionist perspectives that locate problems less in the individual and more in social inequalities and power relations. 
However, it can sometimes be difficult to know the extent to which our clinical judgments are influenced by pragmatic considerations about the availability of resources. Traditionally, we have seen ourselves as clinicians and not as financial managers, but if Lord Layard's proposals to significantly increase access to psychological therapy are implemented, the picture might change altogether. For example, many who may currently appear 'unsuitable' for therapy could become potential candidates for the suggested short term interventions. If we also imagine a world in which will be competing with other therapy service providers, how might this further change our 'clinical' judgments about the suitability of clients for psychological therapy? Judgments made by therapists whose living depends on having enough private therapy clients are likely to experience very different pressures on them, which may lead to more inclusive decisions about 'suitability' than those working in the heavily over-burdened NHS. These observations suggest that we cannot fully divorce the pragmatic from our clinical decisions however much we would like to maintain the 'purity' of our clinical role. This is clearly another inherent conflict that requires ongoing debate and reflection.

\section{Conclusions}

The decision to set up a single point of referral with a dedicated referrals manager and referrals team has been successful. The team is in a good position to ensure that we remain responsive to the dilemmas created by an imbalance of demand and resource without sacrificing the depth and range of work on offer. Upon reflection, we have found that being 'too busy' to think strategically or to respond adaptively simply risks accumulating further long waiting-lists, resulting in distress for clients and clinicians alike.

The changes that have taken place in waiting-list and referrals management echo broader changes in the type of work the department does as well as in its relationship with referrers and colleagues in allied services. There has been a positive shift towards greater openness, collaboration and better communication which has led to greater critical evaluation of departmental functioning and a keener sense of our place on the 'map' of services. This has highlighted the shortfall of local service provision. However, rather than quietly accumulate long lists of clients waiting to be seen, we are now more able to make informed comments about the level of unmet need and to consistently report on the imbalance between demand and resource in the interests of supporting effective, needs-led commissioning.

\section{Acknowledgements}

We would like to acknowledge the huge amount of work by $\mathrm{Dr}$ Philip Dodgson, Dr Brenda Roberts, Ms Nicola Champion, Dr Margie Callanan, the referrals team and the administrative team that has gone into making this referrals system work. We would like to particularly thank Frances Brownrigg for her support with the preparation of the graphs. 


\section{References}

Anderson, K. \& White, J. 1994. 'Evaluation of an opt-in system in primary care psychology.' Clinical Psychology Forum, 93: 28-30.

Ambrose, L. \& Ormond, J. 1994. 'The use of the personal information questionnaire with adults referred to a clinical psychology service.' Clinical Psychology Forum, 93: 31-33.

American Psychiatric Association. 1994. Diagnostic and Statistical Manual of Mental Disorders ( $4^{\text {th }}$ edition), APA, Washington, DC.

Chiesa, M. 1992. 'A comparative study of psychotherapy referrals'. British Journal of Medical Psychology, 65: 5-8.

Core System Group. 1998. CORE System (Information Management) Handbook, Leeds, Core System Group.

Department of Health. 2000. NHS Plan: A Plan for Investment, A Plan for Reform, London, The Stationary Office.

Department of Health. 2004. Organising and delivering psychological therapies, London, The Stationary Office.

Eynon, T. 1993. 'Too high a hurdle? The use of pre-assessment questionnaires in psychotherapy', Psychiatric Bulletin, 17: 149 -151.

Green, B. \& Giblin, M. 1988. 'Screening out non-attenders', Clinical Psychology Forum, 18: 12 -14.

Hughes, I. 1995. 'Why do they stop coming? Reasons for therapy termination by adult clinical psychology clients', Clinical Psychology Forum, 81: $7-12$.

Markman, P. \& Beeney, E. 1990. "DNA rates and the effect of "opting in" to a clinical psychology service', Clinical Psychology Forum, 25: 9 -10.

O'Loughlin, S. 1990. 'The effect of a pre-appointment questionnaire on clinical psychologist attendance rates', British Journal of Medical Psychology, 63: 5 - 9.

Reid, D. \& Mclvor, M. 2005. 'Does client self-booking reduce DNAs in a department of psychological services?', Clinical Psychology Forum, 152: 41- 44.

Rezin, V. \& Gardner, C. 2006. 'A survey of psychological therapy waiting lists in secondary adult mental health services throughout UK NHS Trusts', Clinical Psychology Forum, 163: 30 - 33. 